Proceedings of the 2011 Winter Simulation Conference

S. Jain, R. R. Creasey, J. Himmelspach, K. P. White, and M. Fu, eds.

\title{
FAILURE OF RANDOM MATERIALS: A LARGE DEVIATION AND COMPUTATIONAL STUDY
}

\author{
Jingchen Liu \\ Columbia University \\ 1255 Amsterdam Ave \\ New York, NY 10027, USA \\ Rohit Patra \\ Columbia University \\ 1255 Amsterdam Ave \\ New York, NY 10027, USA
}

\author{
Xiang Zhou \\ Brown University \\ 182, George Street \\ Providence, RI 02912, USA \\ Weinan E \\ Princeton University \\ Fine Hall, Washington Road \\ Princeton, NJ, 08544, USA
}

\begin{abstract}
We study the problem of estimating small failure probabilities for elastic random material described by a one dimensional stochastic elliptic differential equation with certain external forcing and boundary conditions. Gaussian random functions are used to model the spatial variation of the material parameters. The failure event of the bulk material is simply characterized by the exceeding of certain thresholds for the maximum strain in the material. Using large deviation heuristics, we provide an intuitive description of the most probable realization of the random material parameters leading to critical situations of material failure. An efficient Monte Carlo method to compute such probabilities is presented.
\end{abstract}

\section{INTRODUCTION}

When a slowly increasing external load is applied to a composite material, how does the heterogeneous microstructure inside affect the mechanism of fracture and failure of the bulk material? This question is of great importance, technically and theoretically, and an entire branch of material science is devoted to its study (Lawn and Wilshaw 1975). In the structural design of engineering materials, one major problem is the uncertainty in the safety factors for structural strength, i.e., to determine the statistics of the material strength, the maximum load the material can resist before showing macroscopic breakdown.

Physically, material failure is a progressive and localized structural damage that initiates at a microscopic level, controlled by inter-atomic bond breaking, as a result of crack nucleation and growth. It is very hard to simulate the failure process at the most detailed level, in particular when such events have a very small probability to occur. An alternative approach is the probabilistic analysis of the complexity and heterogeneity inherent in materials in the language of random media. In this description, continuum mechanics is still used but macroscopic material behavior is derived in an averaged or "effective" sense. This is the viewpoint taken in homogenization theory (Bensoussan, Lions, and Papanicolaou 1978, Papanicolaou and Varadhan 1982, Jikov, Kozlov, and Oleinik 1994, Pavliotis and Stuart 2008). However, for the study of material failure, which are usually rare events, standard homogenization theory is not enough and the associated central limit theorem (Bal 2008) is not complete and general yet. This note is a tentative step toward an analytical and numerical study of material failure by focusing on a simple one dimensional model problem. 


\section{Liu, Zhou, Patra, and E}

The model we consider is the classical continuum mechanical model in the form of the following elliptic partial differential equation,

$$
\left\{\begin{array}{l}
-\nabla \cdot \sigma(x)=f(x), \\
\sigma(x)=a(x) \nabla u(x) .
\end{array}\right.
$$

The solution to the above equation $u$ is the displacement field of the material, $\nabla u$ is the strain, $\sigma$ is the stress tensor, and $f$ is the external body force. The notation $\nabla \cdot \sigma(x)$ is the divergence of the tensor $\sigma(x)$. The elasticity tensor $a(x)$ (which is positive definite at every point $x$ ) in the constitutive relation $\sigma(x)=a(x) \nabla u(x)$, is determined by the property of the specific material. In this note, we only consider the one dimensional problem. In this case, (1) becomes

$$
\left(a(x) u^{\prime}(x)\right)^{\prime}=-f(x), \quad x \in[0,1],
$$

where $a(x)$ is a positive random function in $\mathbb{C}\left([0,1] \mathbb{R}^{+}\right)$and the force $f$ is deterministic.

The first issue that we have to address is a quantitative measure for failure. It turns out that this is not an easy task and several working definitions are in use in the engineering community. Here we propose one possibile failure criteria, which is consistent with general notions used in the engineering community and which has its own mathematical interest. We define failure as the event that the maximum strain, $\max _{x}|\nabla u(x)|$, exceeds a certain threshold value $b>0$. Our goal is to characterize the probability of failure, i.e., to estimate

$$
p_{b} \triangleq P\left(\max _{x}\left|u^{\prime}(x)\right|>b\right) .
$$

There are several natural ways characterizing the properties of the random field $a(x)$, depending on the specific cases of interests. In this note, we assume that the random function $a$ has log-normal distribution:

$$
a(x)=a_{0}(x) e^{\alpha \xi(x)}
$$

where $a_{0}(x)$ is a deterministic function and $\xi(x)$ is a homogeneous Gaussian random field with zero mean and given covariance. The noise level $\alpha>0$ is assumed to be a constant.

There are two interesting asymptotic regimes. First, for a fixed threshold value $b$, we let $\alpha$ tend to zero. Alternatively, one may fix $\alpha=1$ and let $b$ tend to infinity. Both regimes are interesting under specific scenarios. In this paper, we consider the second regime, that is, sending the threshold value to infinity for a fixed level of noise.

The paper is organized as follows. In the next section, we state our main results on the upper and lower bounds for $p_{b}$. We also discuss an efficient Monte Carlo methods for computing $P\left(\max _{x} u^{\prime}(x)>b\right)$. In Section 3 we present numerical results. The proofs are given in Section 4.

\section{MAIN RESULTS}

\subsection{The Asymptotic Results}

We consider two types of boundary conditions for (2).

1. $u(x=0)=0$ and $\sigma(x=1)=P$ where the load $P$ is a known constant.

2. Dirichlet boundary condition: $u(x=0)=u(x=1)=0$.

The solutions with these boundary conditions are given respectively by:

$$
\begin{aligned}
& u_{1}(x)=\int_{0}^{x} \frac{F(1)-F(y)+P}{a(y)} d y, \\
& u_{2}(x)=\int_{0}^{x} F(y) a^{-1}(y) d y-\int_{0}^{x} a^{-1}(y) d y \frac{\int_{0}^{1} F(y) a^{-1}(y) d y}{\int_{0}^{1} a^{-1}(y) d y},
\end{aligned}
$$




\section{Liu, Zhou, Patra, and E}

where $F(x)=\int_{0}^{x} f(y) d y$. The corresponding strain $u^{\prime}(x)$ is

$$
\begin{aligned}
& u_{1}^{\prime}(x)=a^{-1}(x)(F(1)-F(x)+P), \\
& u_{2}^{\prime}(x)=a^{-1}(x)\left(F(x)-\frac{\int_{0}^{1} F(y) a^{-1}(y) d y}{\int_{0}^{1} a^{-1}(y) d y}\right) .
\end{aligned}
$$

To simplify our discussion, we assume that both $f$ and $P$ are non-negative. For $u_{1}(x)$, the high values of the strain $\max _{x \in[0,1]} u_{1}^{\prime}(x)>b$ are given simply by $\max _{x \in[0,1]} \log u_{1}^{\prime}(x)>\log b$. With $a(x)=a_{0}(x) e^{\xi(x)}$, $\log u_{1}^{\prime}(x)$ is a Gaussian process with spatially varying mean (depending on $a_{0}(x)$ and $F(x)$ ). The asymptotic distribution of the the supremum of a Gaussian process over a compact set has been studied intensively (Adler, Blanchet, and Liu 2011, Adler and Taylor 2007). For the large deviation results on $u_{1}(x)$, which takes the form of an integral of the exponential Gaussian process, see (Liu 2011, Liu and Xu 2011).

In this paper, we focus on the solution to the Dirichlet boundary condition, $u_{2}(x)$. Note that the tail behavior of $\max _{x} u_{2}^{\prime}(x)$ depends very much on the specific form of $F(x)$. We consider one specific case that

$$
F(x)=I\left(x \geq x_{*}\right)
$$

with $x_{*} \in(0,1)$, which corresponds to the situation that a force is applied at one point of the material. To simplify our analysis, we only consider the one-sided tail that

$$
P\left(\max _{x} u_{2}^{\prime}(x)>b\right) .
$$

The analysis of $P\left(\max _{x}\left(-u_{2}^{\prime}(x)\right)>b\right)$ is completely analogous.

The following theorems establish some non-trivial bounds for the quantity in (9).

Theorem 1 Assume that $a(x)=e^{-\xi(x)}$, where $\xi(x)$ is a zero-mean, unit variance, and continuous Gaussian process. Let $u(x)$ be the solution to (2) with boundary condition that $u(0)=u(1)=0$ and $F(x)$ be defined as in (8). Then we have

$$
P\left(\sup _{x \in[0,1]} u^{\prime}(x)>b\right) \leq H e^{-(\log b-a)^{2} / 2}
$$

for some constant $H, a>0$ and all $b>1$.

Theorem 2 Assume that the conditions in Theorem 1 hold. Assume in addition that $\xi(x)$ is a homogeneous and almost surely three times differentiable Gaussian random field such that the covariance function satisfies the local expansion

$$
C(x) \triangleq E(\xi(x) \xi(0))=1-\frac{1}{2} x^{2}+\frac{C_{4}}{24} x^{4}+o\left(x^{4}\right) .
$$

as $x \rightarrow 0$. In addition, assume that $C(x)$ is a monotonically decreasing function on $[0,+\infty)$. Use $Z$ to denote a standard normal random variable. Let

$$
\delta_{*}=\arg \sup _{\delta} P(Z \leq \delta) e^{-\delta^{2} / 2},
$$

and $r$ be defined as

$$
P\left(Z \leq \delta_{*}\right) e^{r-\delta_{*}^{2} / 2}=b
$$

Then, we have the lower bound of $p_{b}$

$$
P\left(\sup _{x \in[0,1]} u^{\prime}(x)>b\right) \geq \varepsilon P(Z>r),
$$

for $b$ sufficiently large and some $\varepsilon>0$. 


\section{Liu, Zhou, Patra, and E}

Remark 1 In the above theorem, we assume that the field $\xi(x)$ is at least three times differentiable, which implies that the covariance function is at least 6 times differentiable. In addition, the first, third, and fifth derivatives of $C(x)$ evaluated at the origin are all zero. The expansion in (10) is of that form up to the first four terms. In addition, we normalize the second derivative $C^{\prime \prime}(0)=-1$, equivalently the spectral moment of order two is unity, which implies that $\operatorname{Var}\left(\xi^{\prime}(x)\right)=1$. This normalization can be obtained by reparameterizing the index $x \in[0,1]$. The constant $C_{4}$ is the spectral moment of order four.

Remark 2 We strongly believe (especially based on the intuition given momentarily) that the lower bound in Theorem 2 is asymptotically sharp in the sense that there exists a constant $H_{*}$ such that

$$
P\left(\sup _{x \in[0,1]} u^{\prime}(x)>b\right)=\left(H_{*}+o(1)\right) P(Z>r) .
$$

This will be pursued in future work.

\subsection{Intuitive Mechanism for the Large Excursions of $u^{\prime}(x)$}

The intuitive interpretation of these two theorems is as follows. The event $\left\{\sup _{x} u^{\prime}(x)>b\right\}$ is very much similar to the event $\left\{\sup _{x} \xi(x)>\log b\right\}$. This is the basis for proving the upper bound in Theorem 1. On the other hand, for $x>x_{*}$, since the factor in (7) satisfies

$$
0<\left(F(x)-\frac{\int_{0}^{1} F(y) a^{-1}(y) d y}{\int_{0}^{1} a^{-1}(y) d y}\right)<1,
$$

it requires that $\sup _{x} \xi(x)$ go above a higher level than $\log b$. Suppose that $\xi(x)$ attains a large value at $\tau \in[0,1]$ with value $\xi(\tau)=r$. Note that $(\xi(x), \xi(\tau))$ is a bivariate normal random vector with zero mean and covariance matrix

$$
\left(\begin{array}{cc}
C(0) & C(x-\tau) \\
C(x-\tau) & C(0)
\end{array}\right) .
$$

Then, the conditional distribution of $\xi(x)$ given $\xi(\tau)=r$ is a normal distribution with mean $r \frac{C(x-\tau)}{C(0)}$ and variance $C(0)\left(1-\left(\frac{C(x-\tau)}{C(0)}\right)^{2}\right)$. Then, using (10), we know that

$$
\xi(x)=E(\xi(x) \mid \xi(\tau)=r)+h(x-\tau)=r C(x-\tau)+h(x-\tau),
$$

where $h(t)$ is a mean-zero Gaussian process and $E\left(h^{2}(t)\right)=1-C^{2}(t)$. Since the variation of $h$ is independent of $r$, we may ignore $h(t)$ and write $\xi(x) \approx r C(x-\tau)$ as $r \rightarrow \infty$. Then, the normalized density

$$
\frac{a^{-1}(x)}{\int_{0}^{1} a^{-1}(y) d y} \approx \frac{\sqrt{r}}{\sqrt{2 \pi}} e^{-\frac{r(x-\tau)^{2}}{2}}
$$

is approximately normal with mean $\tau$ and variance $r^{-1}$. Given that $r \rightarrow \infty$ and that the maximum of $u^{\prime}(x)$ is only attained on the set $\left[x_{*}, 1\right]$, it is necessary to have $\tau$ close to $x_{*}$. Otherwise, if $\tau>x_{*}+\varepsilon$ then the factor in (11) is close to zero (for $x \geq x_{*}$ ); if $\tau<x_{*}-\varepsilon$ then $\sup _{x>x_{*}} \xi(x) \approx r\left(1-\varepsilon^{2} / 2\right) \ll r$. Then, we want to solve for the optimal $\tau$. Using the above heuristic derivation and the rescaling $\tau=x_{*}-\delta / \sqrt{r}$, we approximate the factor as follows

$$
(11) \approx 1-P\left(\tau+Z / \sqrt{r}>x_{*}\right)=P(Z \leq \delta),
$$

which is monotone increasing with $\delta$. 


\section{Liu, Zhou, Patra, and E}

With these heuristic approximations, we now ask: Given that $\max _{x} u^{\prime}(x)>b$, where does $u^{\prime}(x)$ attain its maximum and where does $\xi(x)$ attain its maximum? Because of (11) and the fact that $u^{\prime}(x)$ attains its maximum on the interval $\left[x_{*}, 1\right]$, for each $\delta<0$ (correspondingly $\tau>x_{*}$ ), we have that

$$
\sup _{x \in\left[x_{*}, 1\right]} u^{\prime}(x) \approx e^{r} P(Z \leq \delta) .
$$

which is a monotone increasing function of $\delta$. This suggests that it is more likely to have $\delta>0$ (correspondingly, $\tau<x_{*}$ ). For each $\delta>0$, note that

$$
\sup _{x \in\left[x_{*}, 1\right]} u^{\prime}(x) \approx u^{\prime}\left(x_{*}\right) \approx e^{r-\frac{\delta^{2}}{2}} P(Z \leq \delta) .
$$

Let $\delta_{*}$ be the maximizer of $P(Z \leq \delta) e^{-\delta^{2} / 2}$. We conclude that given $\sup _{x \in[0,1]} u^{\prime}(x)>b$, it is very likely that the maximum of $u^{\prime}(x)$ is attained at (or very close to) $x_{*}$. The global maximum of $\xi(x)$ is likely to be attained at (or very close to) $x=x_{*}-\delta_{*} / \sqrt{r}$ at the level of $r$ which is defined as

$$
e^{r-\delta_{*}^{2} / 2} P\left(Z \leq \delta_{*}\right)=b .
$$

This is how we prove the lower bound in Theorem 2.

\subsection{Computational Method}

We now present an efficient Monte Carlo algorithm for computing

$$
P\left(\max _{x \in[0,1]} u^{\prime}(x)>b\right)
$$

via importance sampling, based on the following change of measure

$$
P(A)=E^{Q}\left(\frac{d P}{d Q} I_{A}\right)
$$

where $Q$ is an alternative measure and the Radon-Nykodym derivative $\frac{d P}{d Q}$ is finite on the set $A$. If the measure $Q$ is well chosen, the variance of $\frac{d P}{d Q} I_{A}$ (under the measure $Q$ ) can be substantially reduced. In particular, if one chooses $Q^{*}(\cdot)=P(\cdot \cap A) / P(A)$ to be the conditional measure given the occurrence of $A$, then $\frac{d P}{d Q} I_{A}$ is almost surely a constant under $Q^{*}$. The measure $Q^{*}$ is usually referred to as the zero-variance change of measure. Therefore, one guide of importance sampling under the setting of rare-event simulation is to design a simulatable and computable change of measure that approximates $Q^{*}$.

In what follows, we present one generic family of change of measures

$$
\frac{d Q}{d P}=\int_{0}^{1} \frac{g(\xi(x))}{\varphi(\xi(x))} h(x) d x
$$

where $\varphi(w)$ is the marginal density function of $\xi(x)$ and $h(x)$ is some density function on $[0,1]$. The above definition of $Q$ is rather abstract. There is another description of $Q$, that is, how one simulates a random field under $Q$. The description of the simulation consists of three steps

1. Generate a random variable $\tau \in[0,1]$ according to density $h(\tau)$.

2. Generate $\xi(\tau)$ according to density $g(\xi(\tau))$.

3. Generate $\{\xi(x): x \neq \tau\}$ according to its original conditional distribution given $(\tau, \xi(\tau))$. 


\section{Liu, Zhou, Patra, and E}

It is not difficult to verify that the above three-step simulation procedure is consistent with the definition of (14). One may have problem-specific choices of the distributions $h$ and $g$. Under the scenario that $\xi(x)$ is homogeneous with mean zero and unit variance, the original density function is

$$
\varphi(w)=\frac{1}{\sqrt{2 \pi}} e^{-\frac{w^{2}}{2}} .
$$

We choose

$$
h(x)=I_{[0,1]}(x), \quad \text { and } g(w)=\frac{1}{\sqrt{2 \pi}} e^{-\frac{w^{2}}{2}} \frac{I(w>\log b-1 / \log b)}{P(Z>\log b-1 / \log b)} .
$$

That is, $h$ is the uniform distribution over $[0,1]$ and $g$ is the conditional distribution of a standard Gaussian random variable given that it exceeds level $\log b-1 / \log b$. Then, with some elementary calculations, the Radon-Nykodym derivative in (14) takes the form

$$
\frac{d Q}{d P}=\frac{m e s\left(A_{\log b-1 / \log b}\right)}{P(Z>\log b-1 / \log b)},
$$

where mes $(\cdot)$ is the Lebesgue measure and $A_{\gamma}=\{x: \xi(x)>\gamma\}$. Note that $d Q / d P>0$ on the set $\left\{\max _{x} \xi(x)>\right.$ $\log b\}$ and $\left\{\max _{x} u^{\prime}(x)>b\right\} \subset\left\{\max _{x} \xi(x)>\log b\right\}$. Therefore, the identity (13) makes sense and our importance sampling estimator for $P\left(\max _{x} u^{\prime}(x)>b\right)$ is

$$
L_{b} \triangleq \frac{P(Z>\log b-1 / \log b)}{m e s\left(A_{\log b-1 / \log b}\right)} I\left(\max _{x} u^{\prime}(x)>b\right) .
$$

To prove that the estimator defined by (16) is indeed "efficient", we need the following result (Adler, Blanchet, and Liu 2011).

Theorem 3 (Theorem 7.5 in Adler, Blanchet, and Liu 2011) Assume that $\xi(x)$ is an almost surely twice differentiable Gaussian process on $[0,1]$. We choose $h$ and $g$ as in (15). Let

$$
L_{b}^{*}=\frac{P(Z>\log b-1 / \log b)}{\operatorname{mes}\left(A_{\log b-1 / \log b}\right)} I\left(\max _{x} \xi(x)>\log b\right) .
$$

Then,

$$
\sup _{b>0} e^{(\log b)^{2}} E^{Q}\left(L_{b}^{*}\right)^{2}<\infty .
$$

Note that $L_{b} \leq L_{b}^{*}$, then $\sup _{b} e^{(\log b)^{2}} E^{Q}\left(L_{b}\right)^{2}<\infty$ is also true. In the literature of rare-event simulation, the efficiency measure is typically based on the so-called relative error. In particular, an estimator $L_{b}$ is said to be weakly efficient in computing probability $p_{b}$ if $E^{Q} L_{b}=p_{b}$ and

$$
\lim _{b \rightarrow \infty} \frac{\log E^{Q} L_{b}^{2}}{2 \log p_{b}}=1
$$

Note that by Jensen's inequlity $E^{Q} L_{b}^{2} \geq\left(E^{Q} L_{b}\right)^{2}$, one always has $\frac{\log E^{Q} L_{b}^{2}}{2 \log p_{b}} \leq 1$. It is sufficient to show that $\liminf _{b \rightarrow \infty} \frac{\log E^{Q} L_{b}^{2}}{2 \log p_{b}} \geq 1$. Thanks to Theorems 2 and 3 , we obtain that for any $\varepsilon>0$,

$$
\lim _{b \rightarrow \infty} \frac{-\log E^{Q} L_{b}^{2}}{-2 \log p_{b}} \geq \lim _{b \rightarrow \infty} \frac{C_{1}+(\log b)^{2}}{C_{2}-2 \log P(Z>r)} \geq \lim _{b \rightarrow \infty} \frac{C_{1}+(\log b)^{2}}{C_{2}-2 \log P(Z>(1+\varepsilon) \log b)}=\frac{1}{(1+\varepsilon)^{2}} .
$$

Therefore, under the conditions of Theorem 2, the estimator $L_{b}$ defined in (16) is weakly efficient in computing $p_{b}=P\left(\max _{x} u^{\prime}(x)>b\right)$. 


\section{Liu, Zhou, Patra, and E}

\section{NUMERICAL RESULTS}

In this section we apply the algorithm discussed to a homogeneous field with mean zero and covariance function

$$
C(x)=e^{-\frac{x^{2}}{2}}
$$

and

$$
F(x)=I(x \geq 0.5)
$$

To implement the algorithm we need to discretize $T$. We let $x_{i}=i / 100$ for $i=1, \ldots, 100$ and $\xi_{i}=\xi\left(x_{i}\right)$. In this situation, the detailed simulation scheme is as follows.

- Generate a random variable $m \sim$ Uniform $\{1,2, \ldots 100\}$

- Simulate $x_{m}$ according to a normal given that it exceeds $\log b-1 / \log b$.

- Simulate $\left\{x_{i}: i \neq m\right\}$ according to the conditional distribution given $\left(m, x_{m}\right)$.

The estimator takes the form that

$$
\frac{P(Z>\log b-1 / \log b)}{\frac{1}{100} \sum_{i=1}^{100} I\left(\xi_{i}>\log b-1 / \log b\right)} I\left(\max _{i} u^{\prime}\left(x_{i}\right)>b\right) .
$$

Note that

$$
u^{\prime}\left(x_{i}\right)=e^{\xi\left(x_{i}\right)}\left(F\left(x_{i}\right)-\frac{\int_{0}^{1} F(y) e^{\xi(y)} d y}{\int_{0}^{1} e^{\xi(y)} d y}\right)
$$

consists of an integral, which we use the Simpson's rule to approximate. We increased the number of discretization points to 1000 and observed that the change of the estimates is within one estimated standard error for $10^{4}$ samples. This is an indication that a discretization of size 100 is enough given the current accuracy level.

The estimated tail probabilities $P\left(\max u^{\prime}(x)>b\right)$ along with the estimated standard deviation are presented in Table 1 based on $10^{4}$ independent simulations. The standard deviation reported in the third column are the estimates of $S D^{Q}\left(L_{b}\right)$. The standard deviation of the estimates reported in the second column equals $S D^{Q}\left(L_{b}\right) / 100$.

Table 1: Numerical results based on $10^{4}$ independent simulations. The standard error reported in the third column is the estimated $S D^{Q}\left(L_{b}\right)$.

\begin{tabular}{cccc}
\hline \hline $\log b$ & Estimate & Std. & Std./Est. \\
\hline 2 & $8.37 \mathrm{E}-03$ & $2.51 \mathrm{E}-02$ & 3.00 \\
3 & $4.07 \mathrm{E}-04$ & $1.51 \mathrm{E}-03$ & 3.70 \\
4 & $7.84 \mathrm{E}-06$ & $3.63 \mathrm{E}-05$ & 4.62 \\
5 & $6.90 \mathrm{E}-08$ & $3.53 \mathrm{E}-07$ & 5.12 \\
\hline
\end{tabular}

\section{PROOFS}

Proof of Theorem 1. According to (7), we have

$$
u^{\prime}(x)=\exp (\xi(x))\left(F(x)-Q_{\xi}\left(Y \geq x_{*}\right)\right),
$$

where $Y$ is a random variable with density function (under $Q_{\xi}$ )

$$
\frac{e^{\xi(y)} I_{[0,1]}(y)}{\int_{0}^{1} e^{\xi(s)} d s}
$$


Note that

$$
P\left(\sup _{x} u^{\prime}(x)>b\right) \leq P\left(\sup _{x} e^{\xi(x)}>b\right)=P\left(\sup _{x} \xi(x)>\log b\right) \leq H e^{-(\log b-a)^{2} / 2},
$$

for all $b>1$ and some positive constant $a$. The last inequality can be obtained by the Borel-TIS inequality (Borell 1975, Tsirelson, Ibragimov, and Sudakov 1976, Adler and Taylor 2007).

Proof of Theorem 2. Let $\tau=x_{*}-\delta_{*} / \sqrt{r}$. It is sufficient to show that there exists $\varepsilon>0$ such that

$$
P\left(\sup u^{\prime}(x)>b \mid \xi(\tau)>r\right) \geq \varepsilon
$$

for all $b>b_{0}$ with $b_{0}$ chosen sufficiently large.

The conditional process. We first provide some calculations of the conditional distribution of $\xi(x)$ given $\left(\xi(\tau), \xi^{\prime}(\tau), \xi^{\prime \prime}(\tau)\right)$. Conditional on $\left(\xi(\tau), \xi^{\prime}(\tau), \xi^{\prime \prime}(\tau)\right)$, we expand the random function

$$
\begin{aligned}
\xi(x) & =E\left[\xi(x) \mid \xi(\tau), \xi^{\prime}(\tau), \xi^{\prime \prime}(\tau)\right]+g(x-\tau) \\
& =\xi(\tau)+\xi^{\prime}(\tau)(x-\tau)+\frac{1}{2} \xi^{\prime \prime}(\tau)(x-\tau)^{2}+g_{3}(x-\tau)+R_{\xi}(x-\tau)+g(x-\tau),
\end{aligned}
$$

where

$$
g_{3}(x-\tau)=\frac{1}{6} E\left[\xi^{\prime \prime \prime}(\tau) \mid \xi(\tau), \xi^{\prime}(\tau), \xi^{\prime \prime}(\tau)\right](x-\tau)^{3} .
$$

Note that $\xi^{\prime \prime \prime}(\tau)$ is independent of $\left(\xi(\tau), \xi^{\prime \prime}(\tau)\right)$ and

$$
E\left[\xi^{\prime \prime \prime}(\tau) \mid \xi(\tau), \xi^{\prime}(\tau), \xi^{\prime \prime}(\tau)\right]=-C_{4} \xi^{\prime}(\tau) .
$$

$g(x)$ is a mean zero Gaussian random field such that $E g^{2}(x)=O\left(|x|^{6}\right)$ as $x \rightarrow 0$. In addition, the distribution of $g(x)$ is independent of $\xi(\tau), \xi^{\prime}(\tau)$, and $\xi^{\prime \prime}(\tau) . R_{\xi}(x-\tau)=\xi(\tau) O\left(|x-\tau|^{4}\right)$ is the remainder term of the Taylor expansion of $E\left[\xi(x) \mid \xi(\tau), \xi^{\prime}(\tau), \xi^{\prime \prime}(\tau)\right]$. In addition, the covariance function can be expanded as

$$
C(x)=1-\frac{1}{2} x^{2}+\frac{C_{4}}{24} x^{4}+R_{C}(x)
$$

where $R_{C}(t)=o\left(|x|^{4}\right)$.

The lower bound. Note that $\left(\xi(\tau), \xi^{\prime}(\tau), \xi^{\prime \prime}(\tau)\right)$ is a mean zero multivariate Gaussian random vector with covariance matrix

$$
\left(\begin{array}{ccc}
1 & 0 & -1 \\
0 & 1 & 0 \\
-1 & 0 & C_{4}
\end{array}\right) .
$$

To simplify notation, we let $w \triangleq \xi(\tau)-r, y \triangleq \xi^{\prime}(\tau)$, and $z \triangleq \xi^{\prime \prime}(\tau)+r$. Conditional on $\xi(\tau)>r$, the overshoot $w$ is asymptotically exponentially distributed with expectation $r^{-1}$. We then localize the event by letting

$$
\mathscr{L}=\left\{|w| \leq A, \quad|y| \leq A, \quad|z| \leq A, \quad \sup _{x}|g(x)| /|x|^{3} \leq A\right\}
$$

for some $A>0$. Note that the set $\mathscr{L}$ in fact depends on $b$. To simplify notation, we omit the index of $b$ in $\mathscr{L}$. It is not hard to show that there exists some $\varepsilon_{0}$ so that

$$
P(\mathscr{L} \mid w>0)>\varepsilon_{0}
$$




\section{Liu, Zhou, Patra, and E}

for all $b$ large enough. Then it is sufficient to provide a lower bound for

$$
P\left(u^{\prime}\left(x_{*}\right)>b, \mathscr{L} \mid w>0\right) .
$$

In what follows, conditional on the specific values of $(w, y, z)$, we consider the probability $P\left(u^{\prime}\left(x_{*}\right)>\right.$ $b, \mathscr{L} \mid w, y, z)$. Note that

$$
u^{\prime}\left(x_{*}\right)=e^{\xi\left(x_{*}\right)}\left(1-Q_{\xi}\left(Y \geq x_{*}\right)\right) .
$$

We first investigate

$$
Q_{\xi}\left(Y \geq x_{*}\right)=\int_{x_{*}}^{1} \frac{e^{\xi(x)}}{\int_{0}^{1} e^{\xi(s)} d s} d x .
$$

We insert the expansions of $\xi(x)$ into the above formula and obtain that on the set $\mathscr{L}$

$$
\begin{aligned}
\xi(x) & =r+w+y(x-\tau)-\frac{r-z}{2}(x-\tau)^{2}+O\left(y|x-\tau|^{3}\right)+O\left(r|x-\tau|^{4}\right) \\
& =r+w+\frac{y^{2}}{2(r-z)}-\frac{r-z}{2}\left(x-\tau-\frac{y}{r-z}\right)^{2}+O\left(y|x-\tau|^{3}\right)+O\left(r|x-\tau|^{4}\right) .
\end{aligned}
$$

To obtain the above expansion, we need the fact that $g(x)=O\left(|x-\tau|^{3}\right)$ on the set $\mathscr{L}$. Therefore, on the set $\mathscr{L}$,

$$
\int_{0}^{1} e^{\xi(s)} d s=\frac{\sqrt{2 \pi}}{\sqrt{(r-z)}} e^{r+w+\frac{y^{2}}{2(r-z)}+O\left(r^{-1}\right)}
$$

Moreover,

$$
\int_{x_{*}}^{1} \frac{e^{\xi(x)}}{\int_{0}^{1} e^{\xi(s)} d s} d x=e^{O\left(r^{-1}\right)} P\left(\tau+\frac{y}{r-z}+Z / \sqrt{r-z}>x_{*}\right),
$$

where $Z$ is a standard normal random variable. For a detailed derivation and the specific form of $O\left(r^{-1}\right)$ in the above display, refer to the work of one of the authors (Liu 2011).

By the condition that $\tau=x_{*}-\delta_{*} / \sqrt{r}$, we have

$$
\int_{x_{*}}^{1} \frac{e^{\xi(x)}}{\int_{0}^{1} e^{\xi(s)} d s} d x=e^{O\left(r^{-1}\right)} P\left(Z>\delta_{*} \sqrt{1-\frac{z}{r}}-\frac{y}{\sqrt{r-z}}\right) .
$$

Therefore, on the set $\mathscr{L}$, we obtain that

$$
\begin{aligned}
F\left(x_{*}\right)-Q_{\xi}\left(Y \geq x_{*}\right) & =e^{O\left(r^{-1}\right)} P\left(Z \leq \delta_{*}-\frac{y}{\sqrt{r-z}}+O\left(r^{-1}\right)\right), \\
& =e^{O\left(r^{-1}\right)} P\left(Z \leq \delta_{*}-\frac{y}{\sqrt{r}}+O\left(r^{-1}\right)\right) .
\end{aligned}
$$

In addition, on the set $\mathscr{L}$, we have that according to (17)

$$
\begin{aligned}
\xi\left(x_{*}\right) & =r+w+y \delta_{*} / \sqrt{r}-\delta_{*}^{2} / 2+O\left(r^{-1}\right) \\
& =r+w-\frac{1}{2}\left(\delta_{*}-y / \sqrt{r}\right)^{2}+O\left(r^{-1}\right) .
\end{aligned}
$$

Lastly, given that

$$
P\left(Z \leq \delta_{*}\right) e^{r-\delta_{*}^{2} / 2}=b,
$$


we obtain that

$$
u^{\prime}\left(x_{*}\right)=e^{r+w-\frac{1}{2}\left(\delta_{*}-y / \sqrt{r}\right)^{2}+O\left(r^{-1}\right)} P\left(Z \leq \delta_{*}-\frac{y}{\sqrt{r}}+O\left(r^{-1}\right)\right) \geq b
$$

if and only if

$$
e^{w-\frac{1}{2}\left(\delta_{*}-y / \sqrt{r}\right)^{2}+O\left(r^{-1}\right)} P\left(Z \leq \delta_{*}-\frac{y}{\sqrt{r}}+O\left(r^{-1}\right)\right) \geq e^{-\delta_{*}^{2} / 2} P\left(Z \leq \delta_{*}\right) .
$$

Define function $h(\delta)=e^{-\delta^{2} / 2} P(Z \leq \delta)$. The left hand side of the above inequality equals to

$$
e^{w} h\left(\delta_{*}-y / \sqrt{r}+O\left(r^{-1}\right)\right) .
$$

Given that $\delta_{*}$ maximizes $h(\delta)$, we have that

$$
h\left(\boldsymbol{\delta}_{*}-y / \sqrt{r}+O\left(r^{-1}\right)\right)=h\left(\boldsymbol{\delta}_{*}\right)+\frac{1}{2} h^{\prime \prime}\left(\boldsymbol{\delta}_{*}\right)\left(y^{2} / r+O\left(r^{-2}\right)\right)=e^{O\left(r^{-1}\right)} h\left(\boldsymbol{\delta}_{*}\right) .
$$

Together with (19), we obtain that, on the set $\mathscr{L}, u^{\prime}\left(x_{*}\right) \geq b$ if and only if $w+O\left(r^{-1}\right) \geq 0$. Note that, conditional on $w>0, r \times w \Rightarrow \operatorname{Exp}(1)$ as $r \rightarrow \infty$ where " $\Rightarrow$ " denotes convergence in distribution. Therefore, for some $\varepsilon$ sufficiently small, we obtain that

$$
P\left(\sup _{x} u^{\prime}(x)>b \mid f(\tau)=r+w>r\right) \geq P\left(u^{\prime}\left(x_{*}\right) \geq b \mid w>0\right)=P(r \cdot w>O(1) \mid \mathscr{L}, w>0) P(\mathscr{L} \mid w>0) \geq \varepsilon .
$$

This concludes the proof.

\section{REFERENCES}

Adler, R., J. Blanchet, and J. Liu. 2011. "Efficient Monte Carlo for high excursions of Gaussian random fields". Accepted by Ann. Appl. Probab. Available via http://arxiv.org/pdf/1005.0812 [accessed October $16,2011]$.

Adler, R., and J. Taylor. 2007. Random fields and geometry. Springer.

Bal, G. 2008. "Central limits and homogenization in random media". Multiscale Model. Simul. 7 (2): $677-702$.

Bensoussan, A., J.-L. Lions, and G. Papanicolaou. 1978. Asymptotic analysis for periodic structures. North Holland.

Borell, C. 1975, June. "The Brunn-Minkowski inequality in Gauss space". Inventiones Mathematicae 30 (2): 207-216.

Jikov, V. V., S. M. Kozlov, and O. A. Oleinik. 1994. Homogenization of differential operators and integral functionals. Springer.

Lawn, B. R., and T. R. Wilshaw. 1975. Fracture of brittle solids. Cambridge solid state science series. Cambridge University Press.

Liu, J. 2011. "Tail Approximations of Integrals of Gaussian Random Fields". Accepted by Ann. Probab. Available via http://arxiv.org/pdf/1006.2837v2 [accessed October 16, 2011].

Liu, J., and G. Xu. 2011. "Some asymptotic results of Gaussian random fields with varying mean functions and the associated processes". Available via http://arxiv.org/pdf/1104.1801 [accessed October 16, 2011].

Papanicolaou, G. C., and S. R. S. Varadhan. 1982. "Boundary value problems with rapidly oscillating coefficients”. In Seria Coll. Math. Soc. János Bolyai 27 (Esztergom, Hungary, 1979), Random fields, 835-873. North-Holland, Amsterdam.

Pavliotis, G., and A. Stuart. 2008. Multiscale methods: Averaging and homogenization. Texts in applied mathematics. Springer.

Tsirelson, B., I. Ibragimov, and V. Sudakov. 1976. "Norms of Gaussian sample functions". In Proceedings of the Third Japan-USSR Symposium on Probability Theory 550:20-41, Tashkent, 1976. 


\section{AUTHOR BIOGRAPHIES}

JINGCHEN LIU is an Assistant Professor in the Department of Statistics at Columbia University. Jingchen received his Ph.D. in statistics from Harvard University in June, 2008. His email address is jcliu@stat.columbia.edu. His research interests cover the probability analysis of approximation of Gaussian random field and efficient Monte Carlo simulation method.

XIANG ZHOU is a post doc in the Division of Applied Mathematics at Brown University. He received his doctorate in applied mathematics from Princeton University in 2009. His email address is xiang_zhou@brown.edu. The focus of his research includes noise-induced transition in non-gradient dynamical systems and importance sampling method for Gaussian field.

ROHIT PATRA is a currently a Ph.D. student in the Department of Statistics at Columbia University. He works on importance sampling method.

WEINAN E is currently a Professor in the Department of Mathematics at Princeton University. His research interests include modeling rare events for physical and chemical problems, multiscale methods for stochastic simulation algorithms and stochastic PDE. 